\title{
Solvable Two-Body Dirac Equation as a Potential Model of Light Mesons ${ }^{\star}$
}

\author{
Askold DUVIRYAK \\ Institute for Condensed Matter Physics of National Academy of Sciences of Ukraine, \\ 1 Svientsitskii Str., UA-79011 Lviv, Ukraine \\ E-mail: duviryak@ph.icmp.lviv.ua
}

Received October 29, 2007, in final form May 07, 2008; Published online May 30, 2008

Original article is available at http://www.emis.de/journals/SIGMA/2008/048/

\begin{abstract}
The two-body Dirac equation with general local potential is reduced to the pair of ordinary second-order differential equations for radial components of a wave function. The class of linear + Coulomb potentials with complicated spin-angular structure is found, for which the equation is exactly solvable. On this ground a relativistic potential model of light mesons is constructed and the mass spectrum is calculated. It is compared with experimental data.
\end{abstract}

Key words: two body Dirac equation; Dirac oscillator; solvable model; Regge trajectories

2000 Mathematics Subject Classification: 81Q05; 34A05

\section{Introduction}

It is well known that light meson spectra are structured into Regge trajectories which are approximately linear and degenerated due to a weak dependence of masses of resonances on their spin state. These features are reproduced well within the exactly solvable simple relativistic oscillator model (SROM) [1, 2, 3] describing two Klein-Gordon particles harmonically bound.

Actually, mesons consist of quarks, i.e., spin-1/2 constituents. Thus potential models based on the Dirac equation are expected to be more appropriate for mesons spectroscopy. In recent years the two body Dirac equations (2BDE) in different formulations ${ }^{1}$ with various confining potentials are used as a relativistic constituent quark models [16, 17, 18, 19, 20, 21, 22, 23, 24, 25, 26]. Some models are universal $[27,28]$, i.e., describe several states of heavy as well as light mesons. The solution of 2BDE is usually obtained by means of perturbative or numerical methods, even in the case of simple potentials. As far as exactly solvable models are concerned, only few examples are known in the literature which represent versions of two-body Dirac oscillator [29, 30, 31, 32, 33]. Similarly to SROM, they all exhibit Regge trajectories which are linear exactly or asymptotically (for large values of angular momentum). But energy levels are spin-dependent, and a type of degeneracy is different from that of SROM and experimental data.

In the present paper we attempt to construct, on the base of $2 \mathrm{BDE}$, the consistent solvable potential model which describes as well as possible actual spectra of light mesons. For this purpose we start from 2BDE with general two-fermion local potential proposed by Nikitin and Fushchich [34]. Following the scheme [35, 7, 28], we perform the radial reduction of general 2BDE and get a set of 8 first-order ODEs, then 4 of first-order ODEs and finally a pair of second-order ODEs. This chain of transformations permit us to write down the set of equations

\footnotetext{
*This paper is a contribution to the Proceedings of the Seventh International Conference "Symmetry in Nonlinear Mathematical Physics" (June 24-30, 2007, Kyiv, Ukraine). The full collection is available at http://www.emis.de/journals/SIGMA/symmetry2007.html

${ }^{1}$ Here we mention three formulations of 2BDE approach. One of them $[4,5,6,7]$ is built as a generalization of the Breit equation [8, 9], two other, $[10,11,12]$ and $[13,14,15]$, originate from Dirac constraint theory.
} 
in a compact form and then to impose constraints for general potential which considerably simplify the equations.

In such a way we find a family of exactly solvable models with linear potentials, which includes two known examples of the Dirac oscillators [29, 31, 32, 33] and new ones. Then we construct integrable extensions of these models describing linear plus Coulomb-like interaction of constituents and giving asymptotically linear Regge trajectories. Free parameters can be used to provide a spin-independent degeneracy for asymptotics of trajectories. It is remarkable that one of the models reproduces exactly the spectrum of SROM. It describes two equal-mass Dirac particles bound by rather non-trivial potential.

Calculated spectra are compared to experimental data for the family of $(\pi-\rho)$-mesons.

The Nikitin-Fushchich anzatz as well as our models are rotary invariant but not Poincaréinvariant, as expected of consistent description of relativistic systems. Thus we propose in Appendix the manifestly covariant equations reduction of which into the center-of-mass reference frame restores the former general equation with the residual rotary symmetry. In this form our models are convenient to compare (and some of them are found equivalent) to other covariant two-body Dirac models known in a literature.

\section{General structure of a potential}

We start with the standard three-dimensional formulation of the two-body Dirac equation. It has the form:

$$
\left\{h_{1}(\boldsymbol{p})+h_{2}(-\boldsymbol{p})+U(\boldsymbol{r})-E\right\} \Phi(\boldsymbol{r})=0,
$$

where $\Phi(\boldsymbol{r})$ is 16-component wave function (Dirac $4 \times 4$-bispinor) of relative position vector $\boldsymbol{r}$,

$$
h_{a}(\boldsymbol{p})=\boldsymbol{\alpha}_{a} \cdot \boldsymbol{p}+m_{a} \beta_{a} \equiv-\mathrm{i} \boldsymbol{\alpha}_{a} \cdot \boldsymbol{\nabla}+m_{a} \beta_{a}, \quad a=1,2,
$$

are Dirac Hamiltonians of free fermions of mass $m_{a}, \boldsymbol{p}=-\mathrm{i} \boldsymbol{\nabla}$, and $U(\boldsymbol{r})$ is an interaction potential. If $\Phi(\boldsymbol{r})$ is presented in $4 \times 4$-matrix representation, the operators $\boldsymbol{\alpha}_{a}$ and $\beta_{a}$ act as follows: $\boldsymbol{\alpha}_{1} \Phi=\boldsymbol{\alpha} \Phi, \boldsymbol{\alpha}_{2} \Phi=\Phi \boldsymbol{\alpha}^{\mathrm{T}}$ etc, where $\boldsymbol{\alpha}$ and $\beta$ are Dirac matrices.

The potential $U(\boldsymbol{r})$ is a multiplication operator in the position representation, which is invariant under spational rotations and inversion. The most general form of such a potential is found in [34]

$$
U(\boldsymbol{r})=\sum_{A=1}^{48} U_{A}(r) \Gamma_{A} .
$$

It is parameterized by 48 arbitrary (complex, in general) functions $U_{A}(r)$ of $r=|\boldsymbol{r}|$ (we will refer to them as partial potentials), and matrices $\Gamma_{A}$ are built in terms of Dirac matrices and unit vector $\boldsymbol{n}=\boldsymbol{r} / r$.

We require of the potential $U$ to be Hermitian with respect to the inner product

$$
\langle\Psi \mid \Phi\rangle=\int d^{3} r \operatorname{Tr}\left(\Psi^{\dagger}(\boldsymbol{r}) \Phi(\boldsymbol{r})\right)
$$

Consequently, the equation (1) becomes Hamiltonian. This requirement reduces an arbitrariness in the general potential (3) to 48 real partial potentials.

Let us construct a Hermitian basis for matrices $\Gamma$ involved in the potential (3). For this purpose we start from the relation for Dirac matrices $\boldsymbol{\alpha}=\gamma^{5} \boldsymbol{\sigma}$, where components $\sigma_{i}(i=1,2,3)$ of the vector $\boldsymbol{\sigma}$ are understood as either Pauli $2 \times 2$-matrices or block-diagonal $4 \times 4$-matrices $\operatorname{diag}\left(\sigma_{i}, \sigma_{i}\right)$. By this $\boldsymbol{\alpha}$ is split into two factors, one of which acts on "particle-antiparticle" 
degrees of freedom while another one - on spin degrees of freedom. We will refer to them as Dirac and spin factors respectively. Similar splitting can be done for arbitrary Dirac operator. Now, taking into account that $\beta$ is scalar, $\gamma^{5}$ is pseudo-scalar, $\boldsymbol{n}$ is vector and $\boldsymbol{\sigma}$ is pseudo-vector with respect to $O(3)$ group, we write down the basis for $\Gamma$-matrices in the product form

$$
\begin{array}{ll}
\Gamma_{\mathrm{ee}}=\left\{I, \beta_{1}, \beta_{2}, \beta_{1} \beta_{2}\right\} \times S, & \Gamma_{\mathrm{oo}}=\gamma_{1}^{5} \gamma_{2}^{5} \times \Gamma_{\mathrm{ee}}, \\
\Gamma_{\mathrm{eo}}=\left\{I, \beta_{1}, \mathrm{i} \beta_{2}, \mathrm{i} \beta_{1} \beta_{2}\right\} \times \gamma_{2}^{5} \times T, & \Gamma_{\mathrm{oe}}=\left.\Gamma_{\mathrm{eo}}\right|_{1 \leftrightarrow 2},
\end{array}
$$

where $I$ is the unit operator, $S$ and $T$ are sets of scalar and pseudo-scalar spin factors

$$
\begin{aligned}
& S=\left\{S_{(i)}, i=1,2,3\right\} \equiv\left\{I, \boldsymbol{\sigma}_{1} \cdot \boldsymbol{\sigma}_{2},\left(\boldsymbol{\sigma}_{1} \cdot \boldsymbol{n}\right)\left(\boldsymbol{\sigma}_{2} \cdot \boldsymbol{n}\right)\right\} \\
& T=\left\{T_{(i)}, i=1,2,3\right\} \equiv\left\{\boldsymbol{\sigma}_{1} \cdot \boldsymbol{n}, \boldsymbol{\sigma}_{2} \cdot \boldsymbol{n},\left(\boldsymbol{n}, \boldsymbol{\sigma}_{1}, \boldsymbol{\sigma}_{2}\right)\right\} .
\end{aligned}
$$

The matrices $\Gamma$ in (5), (6) are grouped as the even-even operators $\Gamma_{\text {ee }}$, odd-odd $\Gamma_{\text {oo }}$, evenodd $\Gamma_{\text {eo }}$ and odd-even operators $\Gamma_{\text {oe }}$, following the Chraplyvy classification [36]. All other $O(3)$-invariant Hermitian matrices built with Dirac matrices and $\boldsymbol{n}$ can be expressed in terms of (5), (6). Correspondingly, the potential (3) can be split into four terms $U_{\mathrm{ee}}, U_{\mathrm{oo}}, U_{\mathrm{eo}}, U_{\mathrm{oe}}$.

It is convenient to use block-vector representation for the wave function instead of matrix one as follows

$$
\Phi(\boldsymbol{r})=\left[\begin{array}{ll}
\Phi_{++}(\boldsymbol{r}) & \Phi_{+-}(\boldsymbol{r}) \\
\Phi_{-+}(\boldsymbol{r}) & \Phi_{--}(\boldsymbol{r})
\end{array}\right] \rightarrow\left[\begin{array}{l}
\Phi_{++}(\boldsymbol{r}) \\
\Phi_{+-}(\boldsymbol{r}) \\
\Phi_{-+}(\boldsymbol{r}) \\
\Phi_{--}(\boldsymbol{r})
\end{array}\right] ;
$$

here $\Phi_{++}(\boldsymbol{r})$ is $2 \times 2$-spinor matrix which represents large-large component of wave function [36] while $\Phi_{+-}(\boldsymbol{r}), \Phi_{-+}(\boldsymbol{r})$ and $\Phi_{--}(\boldsymbol{r})$ are large-small, small-large and small-small components of $\Phi(\boldsymbol{r})$. In this representation the 48 of partial potentials $U_{A}(r)$ in (3) are collected in Dirac multiplets with common spin factors as follows

$$
U=U_{\mathrm{ee}}+U_{\mathrm{oo}}+U_{\mathrm{eo}}+U_{\mathrm{oe}}=\sum_{i=1}^{3}\left(\boldsymbol{\Delta}^{(i)} S_{(i)}+\boldsymbol{\Omega}^{(i)} T_{(i)}\right),
$$

$\boldsymbol{\Delta}=\boldsymbol{\Delta}_{\mathrm{ee}}+\boldsymbol{\Delta}_{\mathrm{oo}}, \boldsymbol{\Omega}=\boldsymbol{\Omega}_{\mathrm{eo}}+\boldsymbol{\Omega}_{\mathrm{oe}}$ (here we omit a superscript $i$ ) and

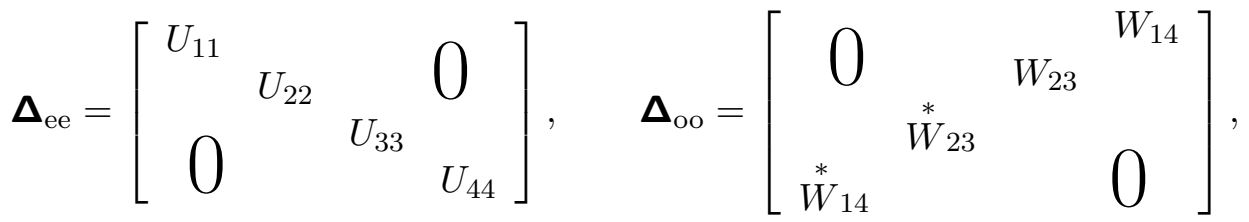

$$
\begin{aligned}
& \boldsymbol{\Omega}_{\mathrm{eo}}=\left[\begin{array}{cccc}
0 & W_{12} & & 0 \\
* & 0 & & 0 \\
W_{12} & 0 & 0 & W_{34} \\
0 & & \stackrel{*}{W} 34 & 0
\end{array}\right], \quad \boldsymbol{\Omega}_{\mathrm{oe}}=\left[\begin{array}{cccc}
0 & & W_{13} & 0 \\
& & 0 & W_{24} \\
W_{13} & 0 & & \\
0 & W_{24}^{*} & 0
\end{array}\right] \text {, }
\end{aligned}
$$

where matrix entries $U_{\alpha \beta}$ are real while $W_{\alpha \beta}=X_{\alpha \beta}+\mathrm{i} Y_{\alpha \beta}$ are complex functions of $r$ (the star "*" denotes a complex conjugation).

\section{Radial reduction of $2 \mathrm{BDE}$}

Following $[35,7,28]$ we choose $\Phi(\boldsymbol{r})$ to be an eigenfunction of the square $\boldsymbol{j}^{2}$ and the component $j_{3}$ of the total angular momentum $\boldsymbol{j}=\boldsymbol{r} \times \boldsymbol{p}+\boldsymbol{s}=-\mathrm{i} \boldsymbol{r} \times \boldsymbol{\nabla}+\frac{1}{2}\left(\boldsymbol{\sigma}_{1}+\boldsymbol{\sigma}_{2}\right)$ and of the parity $P$. In 
a block-matrix representation we have

$$
\begin{aligned}
& \Phi(\boldsymbol{r})=\frac{1}{r}\left[\begin{array}{c}
\mathrm{i} \phi_{1}(r) \mathscr{Y}^{A}(\boldsymbol{n})+\mathrm{i} \phi_{2}(r) \mathscr{Y}^{0}(\boldsymbol{n}) \\
\phi_{3}(r) \mathscr{Y}^{-}(\boldsymbol{n})+\phi_{4}(r) \mathscr{Y}^{+}(\boldsymbol{n}) \\
\phi_{5}(r) \mathscr{Y}^{-}(\boldsymbol{n})+\phi_{6}(r) \mathscr{Y}^{+}(\boldsymbol{n}) \\
\mathrm{i} \phi_{7}(r) \mathscr{Y}^{A}(\boldsymbol{n})+\mathrm{i} \phi_{8}(r) \mathscr{Y}^{0}(\boldsymbol{n})
\end{array}\right] \quad \text { for } P=(-)^{j \pm 1}, \\
& \left(\mathscr{Y}^{A}, \mathscr{Y}^{0}\right) \leftrightarrow\left(\mathscr{Y}^{-}, \mathscr{Y}^{+}\right) \quad \text { for } P=(-)^{j} .
\end{aligned}
$$

Here $\mathscr{Y}^{A}(\boldsymbol{n})$ is an abbreviation of the $2 \times 2$ matrix bispinor harmonics $\mathscr{Y}_{\ell s j}^{\mu}(\boldsymbol{n})$ (in conventional notation $[37,29,27])$ corresponding to a singlet state with a total spin $s=0$ and an orbital momentum $\ell=j$; harmonics $\mathscr{Y}^{0}(\boldsymbol{n}), \mathscr{Y}^{-}(\boldsymbol{n}), \mathscr{Y}^{+}(\boldsymbol{n})$ correspond to triplet with $s=1$ and $\ell=j, j+1, j-1$. Then for $j>0$ the eigenstate problem (1) reduces to the set of eight firstorder differential equations with the functions $\phi_{1}(r), \ldots, \phi_{8}(r)$ and the energy $E$ to be found. Using properties of bispinor harmonics (their explicit form is not important here; see [35, 28] for one)

$$
\begin{aligned}
& \langle i \mid k\rangle=\int d \boldsymbol{n} \operatorname{Tr}\left(\mathscr{Y}_{i}^{\dagger} \mathscr{Y}_{k}\right)=\delta_{i k}, \quad i, k=A, 0,-,+, \\
& j^{2} \mathscr{Y}=j(j+1) \mathscr{Y}, \quad j=0,1, \ldots, \\
& j_{3} \mathscr{Y}=\mu \mathscr{Y}, \quad \mu=-j, \ldots, j, \\
& \ell^{2} \mathscr{Y}=\ell(\ell+1) \mathscr{Y}, \quad \ell=j, j \pm 1, \\
& s^{2} \mathscr{Y}=s(s+1) \mathscr{Y}, \quad s=0,1, \\
& P \mathscr{Y}^{A, 0}=(-)^{j} \mathscr{Y}^{A, 0}, \quad P \mathscr{Y}^{\mp}=(-)^{j \pm 1} \mathscr{Y}^{\mp}, \\
& {\left[\mathscr{Y}^{A}\right]^{\mathrm{T}}=-\mathscr{Y}^{A}, \quad\left[\mathscr{Y}^{0, \mp}\right]^{\mathrm{T}}=\mathscr{Y}^{0, \mp},}
\end{aligned}
$$

and defining a 8-dimensional vector-function $\boldsymbol{\Phi}(r)=\left\{\phi_{1}(r), \ldots, \phi_{8}(r)\right\}$ we present this set in the matrix form

$$
\left\{\mathbf{H}(j) \frac{d}{d r}+\mathbf{V}(r, E, j)\right\} \mathbf{\Phi}(r)=0
$$

Here $8 \times 8$ matrices $\mathbf{H}(j)$ and

$$
\mathbf{V}(r, E, j)=\mathbf{G}(j) / r+\mathbf{m}+\mathbf{U}(r, j)-E \mathbf{I}
$$

possess the properties: $\mathbf{H} \in \operatorname{Re}, \mathbf{H}^{\mathrm{T}}=-\mathbf{H} ; \mathbf{V}^{\dagger}=\mathbf{V} ; \mathbf{I}$ is $8 \times 8$ unity; the diagonal matrix $\mathbf{m}$ and matrices $\mathbf{H}(j), \mathbf{G}(j)$ are constant (i.e. independent of $r$ ), and $\mathbf{U}(r, j)$ represents the potential (3). The operator in the l.h.s. of (12) is Hermitian with respect to the inner product:

$$
\langle\boldsymbol{\Psi} \mid \boldsymbol{\Phi}\rangle=\int_{0}^{\infty} d r\left(\boldsymbol{\Psi}^{\dagger}(r) \boldsymbol{\Phi}(r)\right)
$$

induced by (4). In the case $j=0$ components $\phi_{2}=\phi_{4}=\phi_{6}=\phi_{8}=0$ so that a dimension of the problem (12) reduces from 8 to 4.

Uniting bispinor harmonics in two doublets of opposite parity

$$
\hat{\mathrm{o}}=\left[\begin{array}{l}
\mathscr{Y}^{A} \\
\mathscr{Y}^{0}
\end{array}\right], \quad \hat{\mathrm{e}}=\left[\begin{array}{c}
\mathscr{Y}^{-} \\
\mathscr{Y}^{+}
\end{array}\right]
$$


with the properties

$$
\begin{array}{ll}
\boldsymbol{\sigma}_{1} \cdot \boldsymbol{\sigma}_{2} \hat{\mathrm{o}}=\tau \hat{\mathrm{o}}, & \boldsymbol{\sigma}_{1} \cdot \boldsymbol{\sigma}_{2} \hat{\mathrm{e}}=\hat{\mathrm{e}}, \\
\boldsymbol{\sigma}_{1} \cdot \boldsymbol{n} \hat{\mathrm{o}}=\mathrm{R}^{\mathrm{T}} \hat{\mathrm{e}}, & \boldsymbol{\sigma}_{1} \cdot \boldsymbol{n} \mathrm{e}=\mathrm{R} \hat{\mathrm{o}}, \\
\boldsymbol{\sigma}_{2} \cdot \boldsymbol{n} \hat{\mathrm{o}}=-\sigma_{3} \mathrm{R}^{\mathrm{T}} \hat{\mathrm{e}}, & \boldsymbol{\sigma}_{2} \cdot \boldsymbol{n} \hat{\mathrm{e}}=-\mathrm{R} \sigma_{3} \hat{\mathrm{o}}, \\
\left(\boldsymbol{\sigma}_{1} \cdot \boldsymbol{n}\right)\left(\boldsymbol{\sigma}_{2} \cdot \boldsymbol{n}\right) \hat{\mathrm{o}}=-\sigma_{3} \hat{\mathrm{e}}, & \left(\boldsymbol{\sigma}_{1} \cdot \boldsymbol{n}\right)\left(\boldsymbol{\sigma}_{2} \cdot \boldsymbol{n}\right) \hat{\mathrm{e}}=-\mathrm{R} \sigma_{3} \mathrm{R}^{\mathrm{T}} \hat{\mathrm{o}}, \\
\left(\boldsymbol{n}, \boldsymbol{\sigma}_{1}, \boldsymbol{\sigma}_{2}\right) \hat{\mathrm{o}}=-2 \mathrm{i} \sigma_{\uparrow} \mathrm{R}^{\mathrm{T}} \hat{\mathrm{e}}, & \left(\boldsymbol{n}, \boldsymbol{\sigma}_{1}, \boldsymbol{\sigma}_{2}\right) \hat{\mathrm{e}}=2 \mathrm{i} R \sigma_{\uparrow} \hat{\mathrm{e}},
\end{array}
$$

where $2 \times 2$ matrices $\sigma_{\uparrow}, \sigma_{\downarrow}, \tau$ and $\mathrm{R}$ are defined as follows

$$
\begin{aligned}
& \sigma_{\uparrow}=\frac{1}{2}\left(\mathrm{I}+\sigma_{3}\right)=\left[\begin{array}{ll}
1 & 0 \\
0 & 0
\end{array}\right], \quad \sigma_{\downarrow}=\frac{1}{2}\left(\mathrm{I}-\sigma_{3}\right)=\left[\begin{array}{ll}
0 & 0 \\
0 & 1
\end{array}\right], \\
& \tau=\left[\begin{array}{cc}
-3 & 0 \\
0 & 1
\end{array}\right], \quad \mathrm{R}=\left[\begin{array}{cc}
A & B \\
-B & A
\end{array}\right] \quad(\mathrm{R} \in O(2)), \\
& A=\sqrt{\frac{j+1}{2 j+1}}, \quad B=\sqrt{\frac{j}{2 j+1}}, \quad C=\sqrt{j(j+1)},
\end{aligned}
$$

we present all the matrices involved in the equation (12) in a block-matrix form

$$
\mathbf{m}=\operatorname{diag}\left(m_{+} \mathbf{l}, m_{-} \mathbf{I},-m_{-} \mathbf{l},-m_{+} \mathbf{I}\right)
$$

(here $m_{ \pm}=m_{1} \pm m_{2}$, and $\mathrm{I}$ is a $2 \times 2$ unity),

$$
\begin{aligned}
\mathbf{G} & =-\left[\begin{array}{cccc}
0 & \mathrm{R}\left(j+\sigma_{\uparrow}\right) & \mathrm{R}^{\mathrm{T}}\left(j \sigma_{3}+\sigma_{\uparrow}\right) & 0 \\
\left(j+\sigma_{\uparrow}\right) \mathrm{R}^{\mathrm{T}} & 0 & 0 & \left(j \sigma_{3}+\sigma_{\uparrow}\right) \mathrm{R} \\
\left(j \sigma_{3}+\sigma_{\uparrow}\right) \mathrm{R} & 0 & 0 & \left(j+\sigma_{\uparrow}\right) \mathrm{R}^{\mathrm{T}} \\
0 & \mathrm{R}^{\mathrm{T}}\left(j \sigma_{3}+\sigma_{\uparrow}\right) & \mathrm{R}\left(j+\sigma_{\uparrow}\right) & 0
\end{array}\right], \\
\mathbf{H} & =\left[\begin{array}{cccc}
0 & -\mathrm{R} \sigma_{3} & -\mathrm{R}^{\mathrm{T}} & 0 \\
\sigma_{3} \mathrm{R}^{\mathrm{T}} & 0 & 0 & \mathrm{R} \\
\mathrm{R} & 0 & 0 & \sigma_{3} \mathrm{R}^{\mathrm{T}} \\
0 & -\mathrm{R}^{\mathrm{T}} & -\mathrm{R} \sigma_{3} & 0
\end{array}\right] \text { for } P=(-)^{j \pm 1}, \\
\mathbf{G} & =\left[\begin{array}{cccc}
0 & \left(j+\sigma_{\uparrow}\right) \mathrm{R}^{\mathrm{T}} & \left(j \sigma_{3}+\sigma_{\uparrow}\right) \mathrm{R} & 0 \\
\mathrm{R}\left(j+\sigma_{\uparrow}\right) \sigma_{3} & 0 & 0 & \mathrm{R}^{\mathrm{T}}\left(j \sigma_{3}+\sigma_{\uparrow}\right) \\
\mathrm{R}^{\mathrm{T}}\left(j \sigma_{3}+\sigma_{\uparrow}\right) & 0 & 0 & \mathrm{R}\left(j+\sigma_{\uparrow}\right) \\
0 & \left(j \sigma_{3}+\sigma_{\uparrow}\right) \mathrm{R} & \left(j+\sigma_{\uparrow}\right) \mathrm{R}^{\mathrm{T}} & 0 \\
0 & -\sigma_{3} \mathrm{R}^{\mathrm{T}} & -\mathrm{R} & 0 \\
\mathrm{R} \sigma_{3} & 0 & 0 & \mathrm{R}^{\mathrm{T}} \\
\mathrm{R}^{\mathrm{T}} & 0 & 0 & \mathrm{R} \sigma_{3} \\
0 & -\mathrm{R} & -\sigma_{3} \mathrm{R}^{\mathrm{T}} & 0
\end{array}\right], \text { for } P=(-)^{j} .
\end{aligned}
$$

In order to present the interaction potential (9)-(11) in the block-matrix form we have to calculate in this representation the form of spin operators (7), (8) using the relations (14). One obtains

$$
\begin{array}{lll}
\mathbf{S}=\operatorname{giag}(\mathrm{S}, \Sigma, \Sigma, \mathrm{S}), & \mathbf{T}=\operatorname{giag}\left(\mathbf{T},-\mathrm{T}^{\dagger},-\mathrm{T}^{\dagger}, \mathbf{T}\right) & \text { for } P=(-)^{j \pm 1}, \\
\mathbf{S}=\operatorname{giag}(\Sigma, \mathrm{S}, \mathrm{S}, \Sigma), & \mathbf{T}=\operatorname{giag}\left(\mathbf{T}^{\dagger},-\mathrm{T},-\mathbf{T}, \mathbf{T}^{\dagger}\right) & \text { for } P=(-)^{j},
\end{array}
$$

with the following $2 \times 2$ matrix blocks $S, \Sigma, T$

\begin{tabular}{|c|c|c|c|}
\hline$i$ & 1 & 2 & 3 \\
\hline$S_{(i)}$ & $I$ & $\boldsymbol{\sigma}_{1} \cdot \boldsymbol{\sigma}_{2}$ & $\left(\boldsymbol{\sigma}_{1} \cdot \boldsymbol{n}\right)\left(\boldsymbol{\sigma}_{2} \cdot \boldsymbol{n}\right)$ \\
\hline $\mathrm{S}_{(i)}$ & $\mathrm{I}$ & $\tau$ & $-\sigma_{3}$ \\
\hline$\Sigma_{(i)}$ & $\mathrm{I}$ & $\mathrm{I}$ & $-\mathrm{R} \sigma_{3} \mathrm{R}^{\mathrm{T}}$ \\
\hline
\end{tabular}

\begin{tabular}{|c|c|c|c|}
\hline$i$ & 1 & 2 & 3 \\
\hline$T_{(i)}$ & $\boldsymbol{\sigma}_{1} \cdot \boldsymbol{n}$ & $\boldsymbol{\sigma}_{2} \cdot \boldsymbol{n}$ & $\left(\boldsymbol{n}, \boldsymbol{\sigma}_{1}, \boldsymbol{\sigma}_{2}\right)$ \\
\hline $\mathrm{T}_{(i)}$ & $\mathrm{R}$ & $-\mathrm{R} \sigma_{3}$ & $-2 \mathrm{i} \mathrm{R} \sigma_{\uparrow}$ \\
\hline
\end{tabular}


In the case $j=0$ all $2 \times 2$ blocks in matrices of the equation (12) must be replaced by their left upper entries calculated at $j=0$.

\section{Reduction of 2BDE to a set of second-order ODEs}

It turns out that $\operatorname{rank} \mathbf{H}=4$ (2 for $j=0$ ). In other words, only four equations of the set (12) are differential while remaining ones are algebraic. They can be split by means of an orthogonal (i.e., from the group $O(8)$ ) transformation

$$
\begin{aligned}
& \overline{\mathbf{\Phi}}=\mathbf{O} \boldsymbol{\Phi}, \quad \overline{\mathbf{H}}=\mathbf{O H O}^{\mathrm{T}} \quad \text { etc, } \quad \text { where } \mathbf{O}=\mathbf{O}_{2} \mathbf{O}_{1},
\end{aligned}
$$

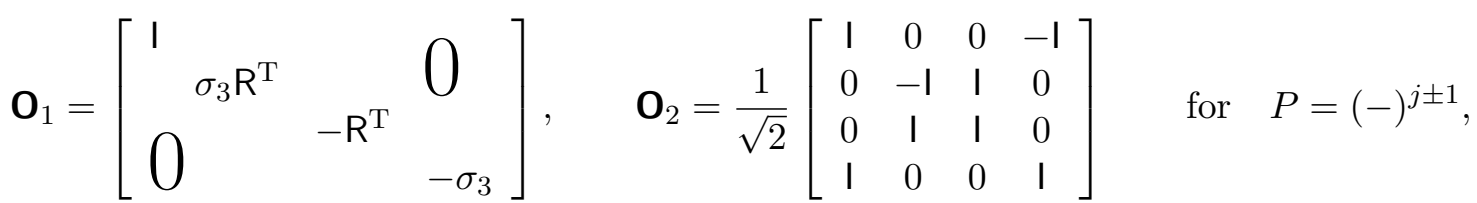

$$
\begin{aligned}
& \mathbf{O}_{1}=\left[\begin{array}{cccc}
\sigma_{3} \mathrm{R}^{\mathrm{T}} & & & 0 \\
& \mathrm{I} & & \\
0 & & -\sigma_{3} & \\
& & -\mathrm{R}^{\mathrm{T}}
\end{array}\right], \quad \mathbf{O}_{2}=\frac{1}{\sqrt{2}}\left[\begin{array}{cccc}
0 & \mathrm{I} & -\mathrm{I} & 0 \\
\mathrm{I} & 0 & 0 & -\mathrm{I} \\
-\mathrm{I} & 0 & 0 & -\mathrm{I} \\
0 & \mathrm{I} & \mathrm{I} & 0
\end{array}\right] \quad \text { for } \quad P=(-)^{j} \text {. }
\end{aligned}
$$

This transformation reduces the matrix $\mathbf{H}$ to the canonical form

$$
\overline{\mathbf{H}}=2\left[\begin{array}{ll}
\mathbf{J} & \mathbf{0} \\
\mathbf{0} & \mathbf{0}
\end{array}\right], \quad \mathbf{J}=\left[\begin{array}{cc}
0 & \mathrm{I} \\
-\mathrm{I} & 0
\end{array}\right],
$$

where $\mathbf{J}$ is a symplectic (nondegenerate) $4 \times 4$ matrix. Other items of (12)-(13) take the form

$$
\begin{aligned}
& \overline{\mathbf{G}}=2\left[\begin{array}{cccc}
0 & \sigma_{\uparrow} & C \sigma_{1} & 0 \\
\sigma_{\uparrow} & 0 & 0 & 0 \\
C \sigma_{1} & 0 & 0 & 0 \\
0 & 0 & 0 & 0
\end{array}\right], \quad \overline{\mathbf{m}}=\left[\begin{array}{cc}
0 & m_{ \pm} \\
-m_{\mp} & 0 \\
m_{ \pm} & 0
\end{array}\right] \quad \text { for } P=\mp\left(-m^{j}\right. \\
& \overline{\mathbf{U}}=\left[\begin{array}{cccc}
\mathrm{U}_{11} & \mathrm{~W}_{12} & \mathrm{~W}_{13} & \mathrm{~W}_{14} \\
\stackrel{W}{12}_{12} & \mathrm{U}_{22} & \mathrm{~W}_{23} & \mathrm{~W}_{24} \\
\mathfrak{*}_{13} & \mathfrak{*}_{23} & \mathrm{U}_{33} & \mathrm{~W}_{34} \\
\stackrel{*}{*}^{*} & \stackrel{*}{*}_{24} & \stackrel{*}{W}_{34} & \mathrm{U}_{44}
\end{array}\right]
\end{aligned}
$$

here $U_{\alpha \beta}$ are real and $W_{\alpha \beta}$ are complex diagonal $2 \times 2$ blocks (related linearly to the former partial potentials $U_{\alpha \beta}, W_{\alpha \beta}$ in (10)-(11)) which are different, in general, for the same $j$ but opposite $P$. Equivalent amount of real partial potentials present in (17) is equal to 32 for $P=(-)^{j \pm 1}$ and 32 for $P=(-)^{j}$, but the only 48 among them are independent.

Let us present 8-dimensional vectors and matrices involved in (12) via 4-dimensional blocks

$$
\overline{\boldsymbol{\Phi}}=\left[\begin{array}{l}
\overline{\boldsymbol{\Phi}}_{1} \\
\overline{\boldsymbol{\Phi}}_{2}
\end{array}\right], \quad \overline{\mathbf{V}}=\left[\begin{array}{ll}
\overline{\mathbf{V}}_{11} & \overline{\mathbf{V}}_{12} \\
\overline{\mathbf{V}}_{21} & \overline{\mathbf{V}}_{22}
\end{array}\right]
$$

and (15) for $\overline{\mathbf{H}}$. In these terms the set (12) splits into differential and algebraic subsets

$$
\begin{aligned}
& 2 \mathbf{J} \overline{\boldsymbol{\Phi}}_{1}^{\prime}+\overline{\mathbf{V}}_{11} \overline{\boldsymbol{\Phi}}_{1}+\overline{\mathbf{V}}_{12} \overline{\boldsymbol{\Phi}}_{2}=0, \\
& \overline{\mathbf{V}}_{21} \overline{\boldsymbol{\Phi}}_{1}+\overline{\mathbf{V}}_{22} \overline{\boldsymbol{\Phi}}_{2}=0 .
\end{aligned}
$$

Eliminating $\overline{\boldsymbol{\Phi}}_{2}$ from (18) by means of (19) yields the purely differential set for 4-vector $\overline{\boldsymbol{\Phi}}_{1}$

$$
\left\{\mathbf{J} \frac{d}{d r}+\mathbf{V}^{\perp}(r, E, j)\right\} \overline{\boldsymbol{\Phi}}_{1}(r)=0 .
$$


The vector $\overline{\boldsymbol{\Phi}}_{2}$ is then determined by the algebraic relation $\overline{\boldsymbol{\Phi}}_{2}=-\mathbf{\Lambda} \overline{\mathbf{V}}_{21} \overline{\boldsymbol{\Phi}}_{1}$. Here

$$
\mathbf{\Lambda}=\left[\overline{\mathbf{V}}_{22}\right]^{-1}, \quad \mathbf{V}^{\perp}=\left(\overline{\mathbf{V}}_{11}-\overline{\mathbf{V}}_{12} \mathbf{\Lambda} \overline{\mathbf{V}}_{21}\right) / 2 .
$$

Next, we present the 4 -vector $\overline{\boldsymbol{\Phi}}_{1}$ in a $2+2$ block form

$$
\overline{\boldsymbol{\Phi}}_{1}(r)=\left[\begin{array}{l}
\Phi_{1} \\
\Phi_{2}
\end{array}\right], \quad \mathbf{V}^{\perp}=\left[\begin{array}{ll}
\mathrm{V}_{11} & \mathrm{~V}_{12} \\
\mathrm{~V}_{21} & \mathrm{~V}_{22}
\end{array}\right], \quad \boldsymbol{\Lambda}=\left[\begin{array}{ll}
\Lambda_{11} & \Lambda_{12} \\
\Lambda_{21} & \Lambda_{22}
\end{array}\right]
$$

then eliminate $\Phi_{2}$ and arrive at the set of second-order differential equations for 2-vector $\Phi_{1}$

$$
\mathrm{L}(E) \Phi_{1} \equiv\left\{\left(\frac{d}{d r}+\mathrm{V}_{12}\right)\left[\mathrm{V}_{22}\right]^{-1}\left(\frac{d}{d r}-\mathrm{V}_{21}\right)+\mathrm{V}_{11}\right\} \Phi_{1}=0
$$

It follows from (13), (16), (17), (21), (22) that the matrix $\mathrm{V}_{22}$ is diagonal. Thus one can perform the transformation

$$
\Psi=\Phi_{1} / \sqrt{\mathrm{V}_{22}}, \quad \tilde{\mathrm{L}}(E)=\sqrt{\mathrm{V}_{22}} \mathrm{~L}(E) \sqrt{\mathrm{V}_{22}},
$$

reducing the equation (23) to a normal form

$$
\tilde{\mathrm{L}}(E) \Psi \equiv\left\{\left(\frac{d}{d r}+\mathrm{F}\right)\left(\frac{d}{d r}-\mathrm{F}^{\dagger}\right)+\mathrm{Z}\right\} \Psi=0
$$

where

$$
\begin{aligned}
& \mathrm{F}=\sqrt{\mathrm{V}_{22}} \mathrm{~V}_{12} / \sqrt{\mathrm{V}_{22}} \text { and } \\
& \mathrm{Z}=\sqrt{\mathrm{V}_{22}} \mathrm{~V}_{11} \sqrt{\mathrm{V}_{22}}+\frac{1}{2}\left(\mathrm{~F} \frac{\mathrm{V}_{22}^{\prime}}{\mathrm{V}_{22}}+\frac{\mathrm{V}_{22}^{\prime}}{\mathrm{V}_{22}} \mathrm{~F}^{\dagger}\right)+\frac{1}{2} \frac{\mathrm{V}_{22}^{\prime \prime}}{\mathrm{V}_{22}}-\frac{3}{4}\left(\frac{\mathrm{V}_{22}^{\prime}}{\mathrm{V}_{22}}\right)^{2} .
\end{aligned}
$$

If $\mathrm{F}$ is Hermitian then the first-order derivative term is absent in the operator $\tilde{L}(E)$ and the equation (24) takes the matrix two-term form

$$
\left\{\frac{d^{2}}{d r^{2}}+\mathrm{Q}\right\} \Psi=0 \quad \text { with } \quad \mathrm{Q}=\mathrm{Z}-\mathrm{F}^{2}-\mathrm{F}^{\prime}
$$

which is useful for a search of solvable examples of 2BDE.

\section{Solvable oscillator-like models}

A construction of the matrix $\mathrm{V}_{22}(22),(21)$ involved in the equations (20), (23), (24) and (26) is a source of non-physical singularities which very complicate an analysis of these equations. Moreover, singularities may make a standard boundary value problem (with constraints at $r=$ $0, \infty$ ) incorrect. We note that in the free-particle case $\mathrm{V}_{22}$ is a constant (free of $r$ ). Let us require of $V_{22}$ to be so in the presence of interaction. Sufficient conditions for this are the equalities

$$
\mathrm{U}_{22}=\mathrm{U}_{33}=\mathrm{W}_{23}=\mathrm{W}_{24}=\mathrm{W}_{34}=0
$$

for entries of the matrix (17) leading to a rather general potential of the form (3) with 18 arbitrary real partial potentials. Four of them compose the even-even part $U_{\text {ee }}$ of the interaction, four other constitute the odd-odd $U_{\mathrm{oo}}$, and the remaining 10 ones form potentials of the $U_{\text {oe }}$ and $U_{\text {eo }}$ type. Due to the constraints (27) both the $U_{\text {ee }}$ and $U_{\text {oo }}$ interaction terms contain the factor $1-\boldsymbol{\sigma}_{1} \cdot \boldsymbol{\sigma}_{2}$. It cancels the interaction on a triplet part of the wave function $\Phi$ and leads to a 
dubious bound state spectrum of poor physical meaning. Thus we discard these potentials by means of the conditions

$$
\mathrm{U}_{11}=\mathrm{U}_{44}=\mathrm{W}_{14}=0
$$

for every of the $P=-(-)^{j}$ and $P=(-)^{j}$ parity cases.

The following requirement

$$
\operatorname{ReW}_{12}=\operatorname{ReW}_{13}=0
$$

causes the equality $\operatorname{Im} F=0$ for $(25)$ and thus $\mathrm{F}^{\dagger}=\mathrm{F}$ (since $\mathrm{F}$ is diagonal, due to the equalities (27)). By this we provide the two-term form (26) for the wave equation. On the other hand ten partial potentials contained in the entries $W_{12}$ and $W_{13}$ of the matrix (17) reduce, due to (28), to 6 ones which form interaction terms of $U_{\text {oe }}$ and $U_{\text {eo }}$ type. Among them the term $U_{0}(r)\left(\boldsymbol{\alpha}_{1}-\boldsymbol{\alpha}_{2}\right) \cdot \boldsymbol{n}$ with an arbitrary function $U_{0}(r)$ is purely gauge and can be compensated by the phase factor $\exp \left\{-\mathrm{i} \int d r U_{0}(r)\right\}$ of $\boldsymbol{\Psi}$. The resulting anzatz for a potential is as follows

$$
\begin{aligned}
U= & \mathrm{i}\left\{U_{1}(r)+U_{2}(r) \gamma_{1}^{5} \gamma_{2}^{5}\right\} \beta_{1} \beta_{2}\left(\boldsymbol{\alpha}_{1}-\boldsymbol{\alpha}_{2}\right) \cdot \boldsymbol{n}+U_{3}(r)\left(\gamma_{1}^{5}+\gamma_{2}^{5}\right)\left(\boldsymbol{\sigma}_{1} \times \boldsymbol{\sigma}_{2}\right) \cdot \boldsymbol{n} \\
& +\mathrm{i}\left\{U_{4}(r) \beta_{1} \gamma_{1}^{5}-U_{5}(r) \beta_{2} \gamma_{2}^{5}\right\}\left(\boldsymbol{\sigma}_{1}-\boldsymbol{\sigma}_{2}+\mathrm{i} \boldsymbol{\alpha}_{1} \times \boldsymbol{\alpha}_{2}\right) \cdot \boldsymbol{n},
\end{aligned}
$$

where $U_{1}(r), \ldots, U_{5}(r)$ are arbitrary real functions.

After aforementioned simplifications the matrices $Q_{+}$and $Q_{-}$involved in the equation (26) and referred to the $P=-(-)^{j}$ and $P=(-)^{j}$ parity cases respectively have the form

$$
\begin{aligned}
\mathrm{Q}_{ \pm}= & \frac{1}{4}\left(E-\frac{m_{+}^{2}}{E}\right)\left(E-\frac{m_{-}^{2}}{E}\right) \\
& -\left[f_{\uparrow \pm}^{2}+\left(\frac{d}{d r}+\frac{2}{r}\right) f_{\uparrow \pm}-\frac{m_{\mp}}{E}\left(2 f_{\uparrow \pm}+\frac{d}{d r}+\frac{2}{r}\right) u_{ \pm}+u_{ \pm}^{2}\right] \sigma_{\uparrow} \\
& -\left[f_{\downarrow}^{2} \pm \frac{d}{d r} f_{\downarrow}\right] \sigma_{\downarrow}-\frac{C}{r}\left[\frac{m_{\mp}}{E}\left(f_{\uparrow \pm} \pm f_{\downarrow}\right)-u_{ \pm}\right] \sigma_{1}-\frac{C^{2}}{r^{2}},
\end{aligned}
$$

where five functions

$$
f_{\uparrow \pm}=\mp\left(U_{1}+U_{2}\right)-2 U_{3}, \quad f_{\downarrow}=U_{2}-U_{1}, \quad u_{ \pm}=-2\left(U_{4} \pm U_{5}\right)
$$

represent equivalently partial potentials. They are a convenient choice when looking for solvable models.

Models I. The choice $u=0$ and $f^{2}=a^{2} r^{2}$, where $a=$ const, leads to four possibilities
Ia) $f_{\uparrow+}=-f_{\uparrow-}=-f_{\downarrow}=-a r$,
Ic) $f_{\uparrow+}=f_{\uparrow-}=f_{\downarrow}=-a r$,
Ib) $f_{\uparrow+}=-f_{\uparrow-}=f_{\downarrow}=-a r$,
Id) $f_{\uparrow+}=f_{\uparrow-}=-f_{\downarrow}=-a r$,

which correspond to the following sets of partial potentials (only non-zero ones are shown)
Ia) $U_{2}=a r$,
Ic) $U_{1}=-U_{2}=U_{3}=\frac{1}{2} a r$,
Ib) $U_{1}=a r$,
Id) $U_{1}=-U_{2}=-U_{3}=-\frac{1}{2} a r$.

All cases lead to the oscillator-like equation

$$
\left\{\frac{d^{2}}{d r^{2}}+\frac{1}{4}\left(E-\frac{m_{+}^{2}}{E}\right)\left(E-\frac{m_{-}^{2}}{E}\right)-a^{2} r^{2}-\frac{C^{2}}{r^{2}}+a \mathrm{D}_{ \pm}\right\} \Psi=0,
$$


where $\mathrm{D}_{ \pm}$is a constant $2 \times 2$ matrix. In the case Ia) this matrix is diagonal,

$$
\text { Ia) } \begin{aligned}
\mathrm{D}_{ \pm} & =\operatorname{diag}\left\{\delta_{\uparrow \pm}, \delta_{\downarrow \pm}\right\}, \\
& \delta_{\uparrow \pm}= \pm 3, \quad \delta_{\downarrow \pm}=\mp 1 \quad \text { for } P=\mp(-)^{j} .
\end{aligned}
$$

The energy spectrum can be found from the algebraic relation

$$
\frac{1}{4}\left(E-\frac{m_{+}^{2}}{E}\right)\left(E-\frac{m_{-}^{2}}{E}\right)=|a|(2 n+3)-a \delta_{\uparrow \pm}, \quad n=j+2 n_{r},
$$

where $n_{r}=0,1, \ldots$ is a radial quantum number. Given $j$ and $n_{r}$ we have 4 equations determining 4 positive values of energy $E_{\uparrow \pm}$

$$
E_{\uparrow \pm}=\sum_{a=1}^{2} \sqrt{m_{a}^{2}+|a|(2 n+3)-a \delta_{\uparrow \pm}} .
$$

In case $\mathrm{Ib}$ ) the matrix $\mathrm{D}_{ \pm}$is not diagonal,

$$
\mathrm{D}_{ \pm}= \pm\left[\begin{array}{cc}
3 & -2 m_{\mp} C / E \\
-2 m_{\mp} C / E & 1
\end{array}\right] \quad \text { for } P=\mp(-)^{j} .
$$

It can be reduced, by means of $O(2)$-transform, to the form (30) with eigenvalues

$$
\text { Ib) } \delta_{\uparrow \pm}=2+\sqrt{1+\left(m_{\mp} C / E\right)^{2}}, \quad \delta_{\downarrow \pm}=2-\sqrt{1+\left(m_{\mp} C / E\right)^{2}} \quad \text { for } P=\mp(-)^{j} \text {. }
$$

The set (29) splits into two oscillator-like equations. Since $\delta$ 's (35) are energy-dependent, the spectral conditions (32) appear in this case as irrational equations which can be reduced to a fourth-order algebraic equations (third-order if $m_{1}=m_{2}$ ) with respect to $E$.

In cases Ic) and Id) the matrix $D_{ \pm}$is equal to either (30)-(31) or (34) for $P=(-)^{j}$ case and to another (complementary) one for opposite parity. The corresponding eigenvalues

$$
\begin{array}{ll}
\text { Ic) } & \delta_{\uparrow+}=2 \pm \sqrt{1+\left(m_{-} C / E\right)^{2}}, \quad \delta_{\uparrow-}=1 \pm 2, \\
\text { Id) } \quad \delta_{\uparrow+}=1 \pm 2, \quad \delta_{\uparrow-}=2 \pm \sqrt{1+\left(m_{+} C / E\right)^{2}}
\end{array}
$$

are to be used in the spectral formulae (32) or (33).

If $m_{1}=m_{2}=0$, the constants $\delta_{\uparrow \pm}$ do not depend on $E$ and $j$ and the equation (32) simplifies to the explicit formula for the energy spectrum

$$
E_{\uparrow \pm}^{2}=4\left[|a|(2 n+3)-a \delta_{\uparrow \pm}\right] .
$$

In this case energy levels $E_{\uparrow \pm}$ build up in the $\left(E^{2}, j\right)$-plane into parallel straight lines (so called Regge trajectories) with the slope rate $8|a|$. All the trajectory can be labeled unambiguously by the triplet of quantum numbers $n_{r}, \uparrow, \pm$ but some trajectories corresponding to different numbers may coincide (i.e., degenerate). Examples of Regge trajectories for massless models Ia) - Id) are shown in the Fig. 1.

Models II. The choice $f_{\uparrow}=0$ and $f_{\downarrow}^{2}=u^{2}=a^{2} r^{2}$ leads to four possibilities

$$
\begin{array}{ll}
\text { IIa) } \quad f_{\downarrow}= \pm u_{ \pm}=-a r, & \text { IIc) } \quad f_{\downarrow}=u_{ \pm}=-a r, \\
\text { IIb) } f_{\downarrow}=\mp u_{ \pm}=-a r, & \text { IId }) \quad f_{\downarrow}=-u_{ \pm}=-a r,
\end{array}
$$

which correspond to the following sets of partial potentials (only non-zero ones are shown)
IIa) $U_{1}=U_{4}=\frac{1}{2} a r$
IIc) $U_{1}=U_{5}=\frac{1}{2} a r$,
IIb) $U_{1}=-U_{4}=-\frac{1}{2} a r$,
IId) $U_{1}=-U_{5}=-\frac{1}{2} a r$. 

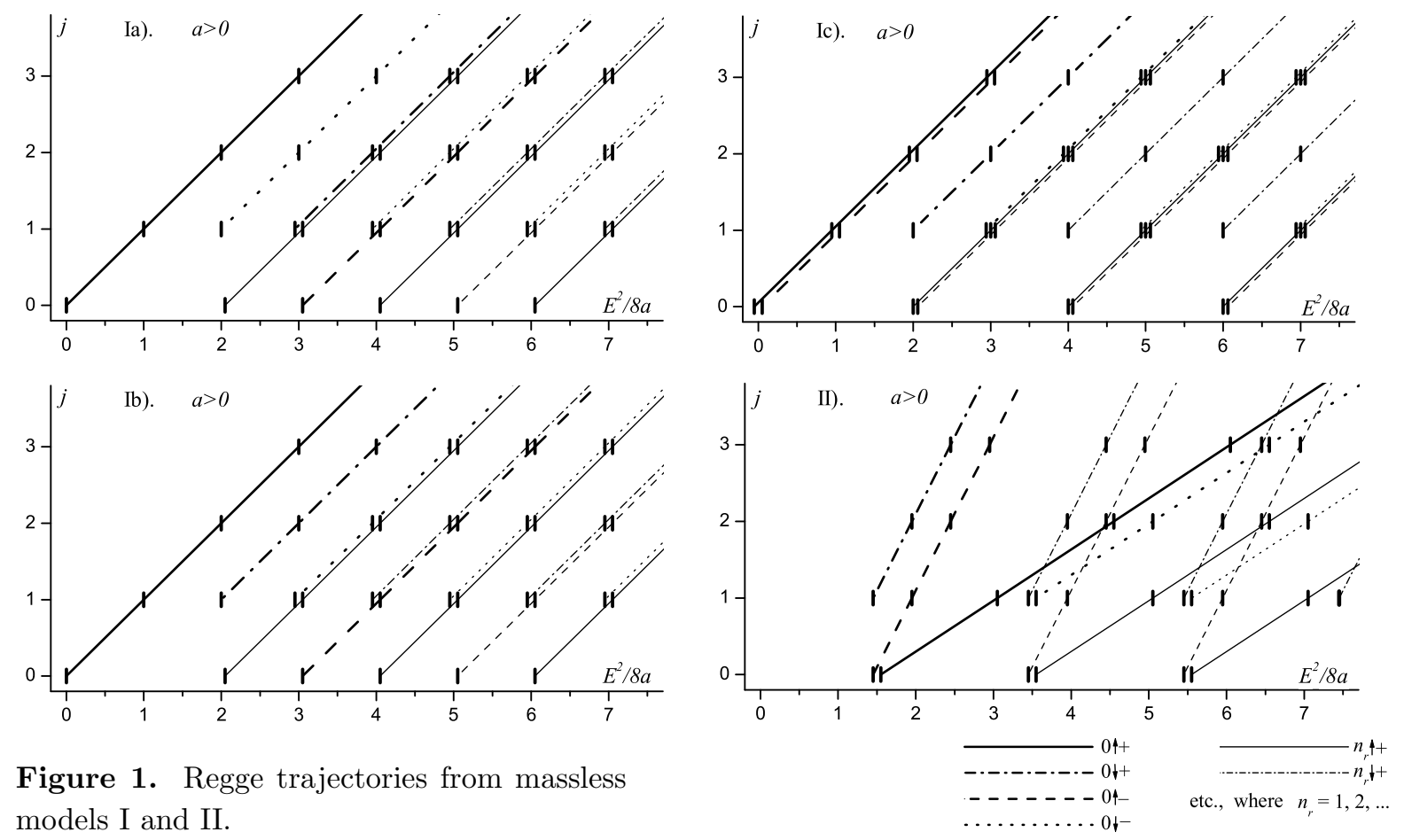

Figure 1. Regge trajectories from massless models I and II.

This choice leads to four solvable models described by the oscillator-like equation (29) with some matrices D. Here we consider the massless case only. All models IIa)-IId) have the same spectrum

$$
\begin{array}{ll}
a>0: & a<0: \\
E_{\uparrow+}^{2}=4 a\left(3 j+4 n_{r}+3\right), & E_{\uparrow+}^{2}=4|a|\left(j+4 n_{r}+3\right), \\
E_{\downarrow+}^{2}=4 a\left(j+4 n_{r}+2\right), & E_{\downarrow+}^{2}=4|a|\left(3 j+4 n_{r}+4\right), \\
E_{\uparrow-}^{2}=4 a\left(j+4 n_{r}+3\right), & E_{\uparrow-}^{2}=4|a|\left(3 j+4 n_{r}+3\right), \\
E_{\downarrow-}^{2}=4 a\left(3 j+4 n_{r}+4\right), & E_{\downarrow-}^{2}=4|a|\left(j+4 n_{r}+2\right) .
\end{array}
$$

In contrast to models I, here the Regge trajectories are not parallel: two families are sloped by $4|a|$, two other - by $12|a|$ (see Fig. 1).

Models III. The conditions $f_{\uparrow}-=u_{+}=0, f_{\uparrow+}^{2}=f_{\downarrow}^{2}=u_{-}^{2}=a^{2} r^{2}$ yield four solvable models one example of which corresponds to the potentials

$$
U_{1}=\frac{3}{4} a r, \quad U_{2}=U_{5}=-\frac{1}{4} a r, \quad U_{3}=U_{4}=\frac{1}{4} a r .
$$

These models (in the massless case) lead to sequences of Regge trajectories with three different slopes: $4|a|, 8|a|$ and $12|a|$.

Two of the above models are known in the literature. Model Ia) is equivalent to the Sazdjian pseudo-scalar confinement model [29]; it coincides with one of versions of two-body Dirac oscillator proposed in [32]. Supersymmetric aspect of these models has been studied in [30, 33]. Model $\mathrm{Ib}$ ) is a generalization of another version of Dirac oscillator [31] to the case of different rest masses.

While having no direct physical meaning, the energy eigenvalues of the models possess some important features of actual meson spectra which we discuss in the next section. 


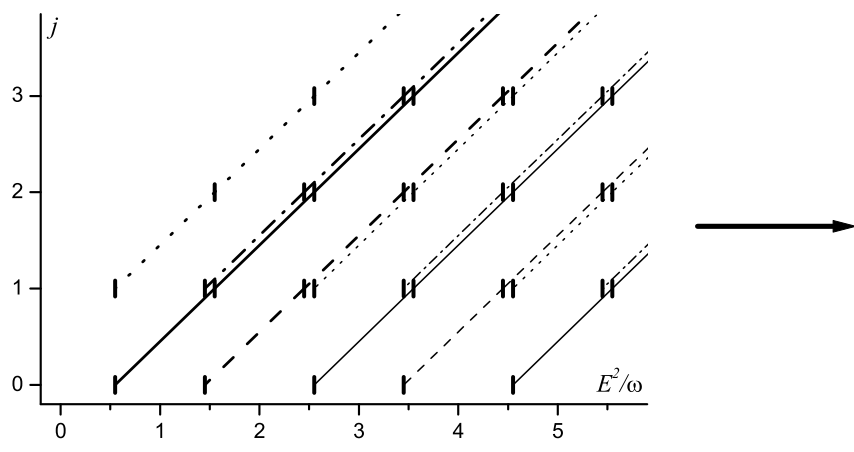

Figure 2. Regge trajectories of idealized light meson spectra; $\kappa=2, \zeta=1 / 2$.

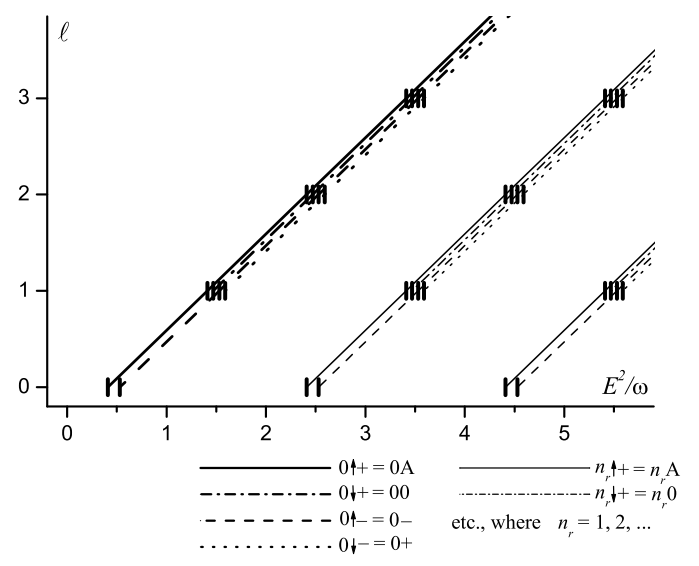

\section{$6 \quad$ Light meson spectra}

Characteristic features of mass spectra of light mesons (consisting of $\mathrm{u}, \mathrm{d}$ and $\mathrm{s}$ quarks) can be summarized roughly in the following idealized picture [38, 39, 7, 28]:

1. Meson states are clustered in the family of straight lines in the $\left(E^{2}, j\right)$-plane known as Regge trajectories.

2. Regge trajectories are parallel; slope parameter $\omega$ is an universal quantity, $\omega=1.15 \mathrm{GeV}^{2}$.

3. Mesons can be classified non-relativistically, as $\left(n^{2 s+1} \ell_{j}\right)$-states of quark-antiquark system ( $n=n_{r}+\ell+1$ where $\ell$ and $n_{r}$ are the orbital and radial quantum number).

4. Spectrum is $\ell s$-degenerated, i.e., masses are distinguished by $\ell$ (not by $j$ or $s$ ) and $n_{r}$.

5. States of different $\ell$ and $n_{r}$ possess an accidental degeneracy which causes a tower structure of the spectrum.

It follows from items $1-3$ that there exist 4 principal $\left(n_{r}=0\right)$ trajectories, one of which includes singlet states $(s=0, j=\ell)$, and three others collect different triplet states $(s=1, j=$ $\ell, \ell \pm 1)$. Each of principal trajectories heads a sequence of daughter trajectories $\left(n_{r}=1,2, \ldots\right)$. Item 4 means that in the $\left(E^{2}, \ell\right)$-plane the four parents degenerate completely (this is concerned also with daughters of the same $n_{r}$ ). Then energy levels of $\mathrm{q}-\overline{\mathrm{q}}$ system can be described by a formula

$$
E^{2} \approx \omega\left(\ell+\kappa n_{r}+\zeta\right)
$$

where a constant $\zeta$ depends on a flavor content of mesons $(\zeta \approx 1 / 2$ for $(\pi$ - $\rho)$-family of mesons; it grows together with quark masses). Finally, item 5 restricts the constant $\kappa$ to an integer or rational number [39]. One frequently puts $\kappa=2$ (oscillator-like degeneracy) [1, 2, 3, 40], rarely $\kappa=1$ (Coulomb-like one) [41, 42]. Fig. 2 illustrates the case of $\left(\ell+2 n_{r}\right)$-degeneracy where corresponding Regge trajectories are plotted in planes $\left(E^{2}, j\right)$ and $\left(E^{2}, \ell\right)$.

Actual spectra of light mesons differ from the idealized spectrum (36). A finite number of mesons are known, some meson states are ambiguously identified by quantum numbers, Regge trajectories are not quite straight (especially, in their bottom) [43] degeneracy is approximated $(\sim 5 \div 6 \%$ of $\omega)$ etc. Thus the equation (36) represents light meson spectra as a rather rough approximation, and its subsequent correction is implied.

Most of features described above are characteristic of spectra of the models Ia)-Id) (see Section 5). Indeed, in the massless case Regge trajectories are straight and parallel. The trajectories $\uparrow+$ consisting of energy levels $E_{\uparrow+}$ of parity $P=-(-)^{j}$ can be treated as a singlet trajectories corresponding to $s=0, \ell=j$ (and labeled by "A"; see Section 3). Similarly, triplet 
quantum numbers $s=1, \ell=j, j \mp 1$ can be prescribed to the trajectories $\downarrow+, \uparrow-$ (labeled by "0", “干"). In these terms the spectrum of the equal-mass $\left(m_{1}=m_{2} \equiv m\right)$ model Ia) is as follows:

$$
E^{2}=8|a|\left(\ell+2 n_{r}+3 / 2\right)+8 a(2 s-3 / 2)+4 m^{2}
$$

which agrees with results presented in $[29,30,32,33]$. For the model Ic) we have

$$
E^{2}=8|a|\left(\ell+2 n_{r}+3 / 2\right)+8 a(s-3 / 2)+4 m^{2}
$$

(the only difference consists in a coefficient at $s$ ).

All models Ia)-Id) reveal degenerated trajectories. But the degeneracy is not a $\ell s$ one. In order to provide $\ell s$-degeneracy one should have a model possessing additional $O(3)$-symmetry with corresponding conserved generators [44]. The total spin $s=\frac{1}{2}\left(\boldsymbol{\sigma}_{1}+\boldsymbol{\sigma}_{2}\right)$ does not match for this role since even the free two-body Dirac Hamiltonian does not commute with $\boldsymbol{s}$. Below we propose generalizations of the I-type models which reveals an approximated $\ell s$-degeneracy at some values of free parameters.

Model IV is an integrable extension of the model $\mathrm{Ib})$. We put $u=0$ and

$$
f_{\uparrow \pm}=\mp a r+\left(\varkappa_{ \pm}-1\right) / r, \quad f_{\downarrow}=-a r+\chi / r,
$$

where $\varkappa_{+}, \varkappa_{-}$and $\chi$ are arbitrary constants. So the model includes, together with a slope parameter $a$ and rest particle masses $m_{1}, m_{2}$, six arbitrary parameters.

The choice (37) corresponds to the following partial potentials

$$
U_{1}=a r-\frac{\varkappa_{+}-\varkappa_{-}+2 \chi}{4 r}, \quad U_{2}=-\frac{\varkappa_{+}-\varkappa_{-}-2 \chi}{4 r}, \quad U_{3}=-\frac{\varkappa_{+}+\varkappa_{-}-2}{4 r} .
$$

The matrix $Q_{ \pm}$in the wave equation (26) has the form

$$
\begin{aligned}
\mathrm{Q}_{ \pm}= & \frac{1}{4}\left(E-\frac{m_{+}^{2}}{E}\right)\left(E-\frac{m_{-}^{2}}{E}\right)-a^{2} r^{2}+a(2 \chi \pm 1)-\frac{C^{2}+\chi(\chi \pm 1)}{r^{2}} \\
& +2\left( \pm a-\frac{\varkappa_{ \pm} \pm \chi-1}{2 r^{2}}\right)\left[\begin{array}{cc}
\varkappa_{ \pm} \mp \chi & -m_{\mp} C / E \\
-m_{\mp} C / E & 0
\end{array}\right] .
\end{aligned}
$$

The matrix in r.h.s. of (39) and thus the $\mathrm{Q}_{ \pm}$itself can be diagonalized, similarly to case Ib). Then the equation (26) splits into two oscillator-like equations with $j$ - and $E$-dependent free term and coefficient at $1 / r^{2}$ term. Spectral conditions which in general are cumbersome irrational equations cannot be solved explicitly. In a massless case we get exact expressions for Regge trajectories

$$
\begin{array}{ll}
E_{\uparrow \pm}^{2}=8|a|\left(k_{\uparrow \pm}+2 n_{r}+\frac{3}{2}\right) \mp 8 a\left(\varkappa_{ \pm}+\frac{1}{2}\right), & k_{\uparrow \pm}=\sqrt{\left(j+\frac{1}{2}\right)^{2}+\varkappa_{ \pm}\left(\varkappa_{ \pm}-1\right)}-\frac{1}{2}, \\
E_{\downarrow \pm}^{2}=8|a|\left(k_{\downarrow \pm}+2 n_{r}+\frac{3}{2}\right)-8 a\left(\chi \pm+\frac{1}{2}\right), & k_{\downarrow \pm}=\sqrt{\left(j+\frac{1}{2}\right)^{2}+\chi(\chi \pm 1)}-\frac{1}{2} .
\end{array}
$$

The Coulomb-like terms in the potentials (38) bend Regge trajectories (especially in the bottom) leaving their asymptotics $($ at $j \rightarrow \infty)$ rectilinear (since $k_{\uparrow \pm} \stackrel{j \rightarrow \infty}{\approx} j+O(1 / j)$ ). When choosing $\varkappa_{+}=\chi, \varkappa_{-}=-\chi-2$ (where $\chi$ is still arbitrary), trajectories become degenerated asymptotically in the plane $\left(E^{2}, \ell\right)$. This model satisfies approximately item 4 of the properties of meson spectra but leads to top-heavy meson masses.

Model $\mathbf{V}$ is another extension of $\mathrm{Ib}$ ) with the same amount of free parameters as in model IV. We put

$$
u_{ \pm}=2 \varkappa_{ \pm} / r, \quad f_{\uparrow \pm}=\mp\{a r-(\chi \mp 1) / r\}, \quad f_{\downarrow}=-a r+\chi / r,
$$


which correspond to the potentials

$$
U_{1}=a r-\frac{\chi}{r}, \quad U_{2}=0, \quad U_{3}=\frac{1 / 2}{r}, \quad U_{4}=-\frac{\varkappa_{+}+\varkappa_{-}}{2 r}, \quad U_{5}=-\frac{\varkappa_{+}-\varkappa_{-}}{2 r} .
$$

The matrix $Q_{ \pm}$is as follows

$$
\begin{aligned}
\mathrm{Q}_{ \pm}= & \frac{1}{4}\left(E-\frac{m_{+}^{2}}{E}\right)\left(E-\frac{m_{-}^{2}}{E}\right)-a^{2} r^{2}+a(2 \chi \pm 1)-\frac{C^{2}+\chi(\chi \pm 1)}{r^{2}} \\
& -2\left\{ \pm \frac{m_{\mp}}{E}\left(a-\frac{\chi \mp 1 / 2}{r^{2}}\right)+\frac{\varkappa_{ \pm}}{r^{2}}\right\}\left[\begin{array}{cc}
2 \varkappa_{ \pm} & C \\
C & 0
\end{array}\right] .
\end{aligned}
$$

Again, an evident diagonalization of $Q_{ \pm}$leads to a split pair of oscillator-like equations and spectral conditions similar to case IV. In a massless case we have the spectrum

$$
E_{\uparrow}^{2}=8|a|\left(k_{\uparrow}+2 n_{r}+\frac{3}{2}\right)-8 a\left(\chi \pm \frac{1}{2}\right),
$$

where

$$
k_{\uparrow}=\sqrt{C^{2}+\left(\chi \mp \frac{1}{2}\right)^{2}+2|\varkappa|\left(|\varkappa| \pm \sqrt{C^{2}+\varkappa^{2}}\right)}-1 / 2 \stackrel{j \rightarrow \infty}{\approx} j \pm|\varkappa|+O(1 / j)
$$

and parity indexes " \pm " are implied. Regge trajectories are asymptotically linear but there is no a choice of parameters providing the $\ell s$-degenegacy.

Models IV and V represent maximal solvable extensions of model Ib) found in this work. There exist extensions of other model of family I which reflect several features of light meson spectra but are exactly solvable with less number of free parameters than models IV and V. Here we consider one example which has close relevance to a meson spectroscopy.

Model VI is the integrable extension of model Ic). We put $u=0$ and

$$
f_{\uparrow+}=-a r+(\varkappa-1) / r, \quad f_{\uparrow-}=f_{\downarrow}=-a r+\chi / r,
$$

which corresponds to the potentials

$$
\begin{array}{ll}
U_{1} & =\frac{1}{2}\left(a r-\frac{\varkappa+\chi-1}{2 r}\right), \quad U_{2}=-\frac{1}{2}\left(a r+\frac{\varkappa-3 \chi-1}{2 r}\right), \\
U_{3} & =\frac{1}{2}\left(a r+\frac{\varkappa+\chi-1}{2 r}\right) .
\end{array}
$$

The matrix $Q_{+}$is identical to that (39) of model IV and a treatment is the same. The matrix $Q_{-}$ in the present case is diagonal

$$
\begin{aligned}
& Q_{-}=\operatorname{diag}\left\{Q_{\uparrow-}, Q_{\downarrow-}\right\}, \\
& Q_{\downarrow-}=\frac{1}{4}\left(E-\frac{m_{+}^{2}}{E}\right)\left(E-\frac{m_{-}^{2}}{E}\right)-a^{2} r^{2}+a\{2(\chi \pm 1)+1\}-\frac{C^{2}+\chi(\chi+1)}{r^{2}} .
\end{aligned}
$$

In the case of equal particle masses, $m_{1}=m_{2} \equiv m$, we get the energy spectrum explicitly

$$
\begin{array}{ll}
E_{\uparrow+}^{2}=8|a|\left(k_{\uparrow+}+2 n_{r}+\frac{3}{2}\right)-8 a\left(\varkappa+\frac{1}{2}\right)+4 m^{2}, & k_{\uparrow+}=\sqrt{\left(j+\frac{1}{2}\right)^{2}+\varkappa(\varkappa-1)}-\frac{1}{2}, \\
E_{\downarrow+}^{2}=8|a|\left(k_{\downarrow+}+2 n_{r}+\frac{3}{2}\right)-8 a\left(\chi+\frac{1}{2}\right)+4 m^{2}, & k_{\downarrow+}=\sqrt{\left(j+\frac{1}{2}\right)^{2}+\chi(\chi-1)}-\frac{1}{2},(40) \\
E_{\uparrow-}^{2}=8|a|\left(k_{\uparrow-}+2 n_{r}+\frac{3}{2}\right)-8 a\left(\chi+\frac{1}{2} \pm 1\right)+4 m^{2}, & k_{\uparrow-}=\sqrt{\left(j+\frac{1}{2}\right)^{2}+\chi(\chi+1)}-\frac{1}{2} .
\end{array}
$$

Regge trajectories are asymptotically linear and, if $\varkappa=\chi, \ell s$-degenerated (see Fig. 3). 

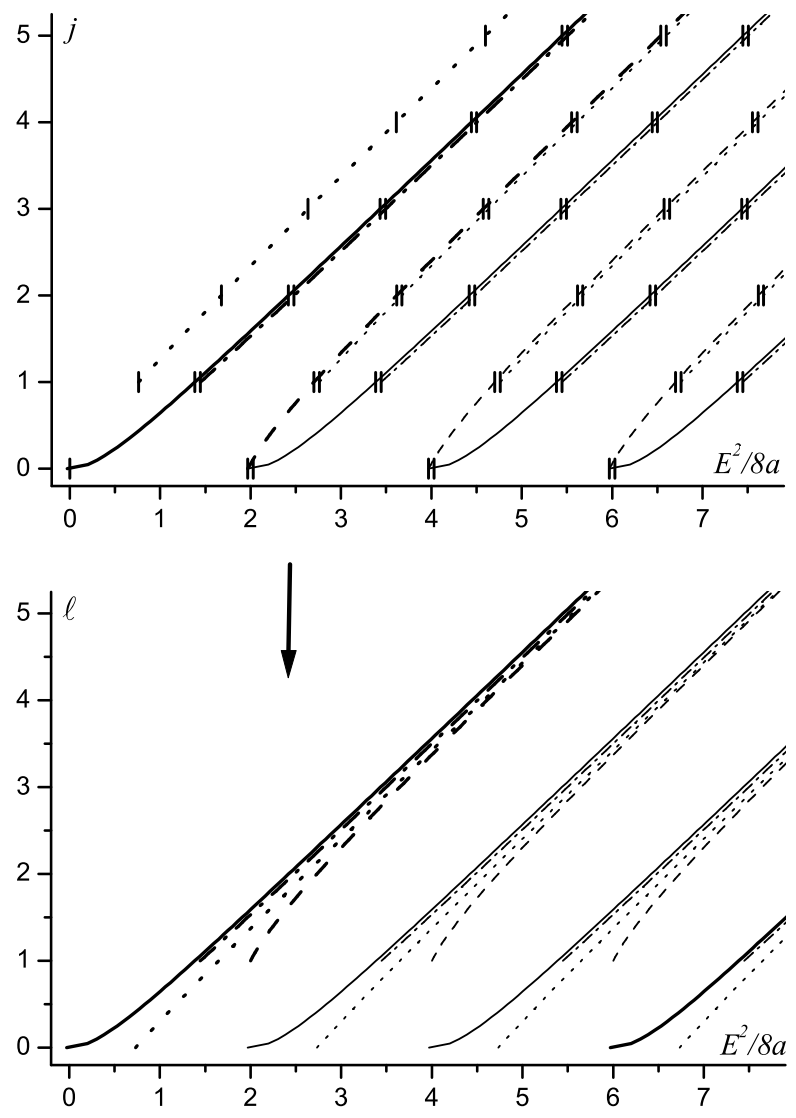

Figure 3. Regge trajectories from model VI; $m_{1}=m_{2}=0, \chi=\varkappa=1 / 2$.

Let us try to describe the spectrum of light mesons of the $(\pi-\rho)$-family by means of equations (40) using four arbitrary parameters $a, \chi, \varkappa$ and $m$ as adjustable parameters. We note that the intersection of the $\rho(770)$-trajectory (which is $0 \downarrow-$ in our terms) with the $E^{2}$-axis in the $\left(E^{2}, j\right)$-plane is negative while it follows from $(40): E_{\downarrow-}^{2}\left(j=0, n_{r}=0\right) \geq 4 m^{2}$. On the whole one can achieve a qualitative and partially numerical agreement of the model with experimental data when supposing that the meson masses squared $M^{2}$ are related to $E^{2}$ as follows: $M^{2}=E^{2}-c^{2}$ where the parameter $c^{2}>0$ is common for all states ${ }^{2}$. Similar picture arises within other models, in particular, in [24] where the meaning of the constant $c$ is discussed. Alternatively, one supposes $m^{2}<0$ (instead of the use of $c$ ) in some SROM potential models [2, 45]. This is not acceptable here since imaginary rest masses break the Hermicity of the two-body Dirac Hamiltonian (1).

It is shown in Fig. 4 the spectrum of the $(\pi-\rho)$-family plotted with the data [46] and corresponding Regge trajectories built with optimal values of parameters: $a=0.145 \mathrm{GeV}^{2}, \chi=0.227$, $\varkappa=0.342, c^{2}=0.346 \mathrm{GeV}^{2}$. Principal trajectories fit majority of meson states. Some radially excited levels, however, are situated far from daughter trajectories.

In the case $\varkappa=\chi=0$ trajectories become linear and degenerated exactly, and formulae (40) drastically simplify

$$
E^{2}=8|a|\left(\ell+2 n_{r}+\frac{3}{2}\right)+4\left(m^{2}-a\right) .
$$

Except for a definition of an additive constant this formula describes exactly the spectrum of the relativistic system of two spinless particles harmonically bound (SROM) $[1,2,3]$. In the

\footnotetext{
${ }^{2}$ The parameter $c^{2}$ absorbs in (40) the term $4 m^{2}$ which then is set to zero.
} 


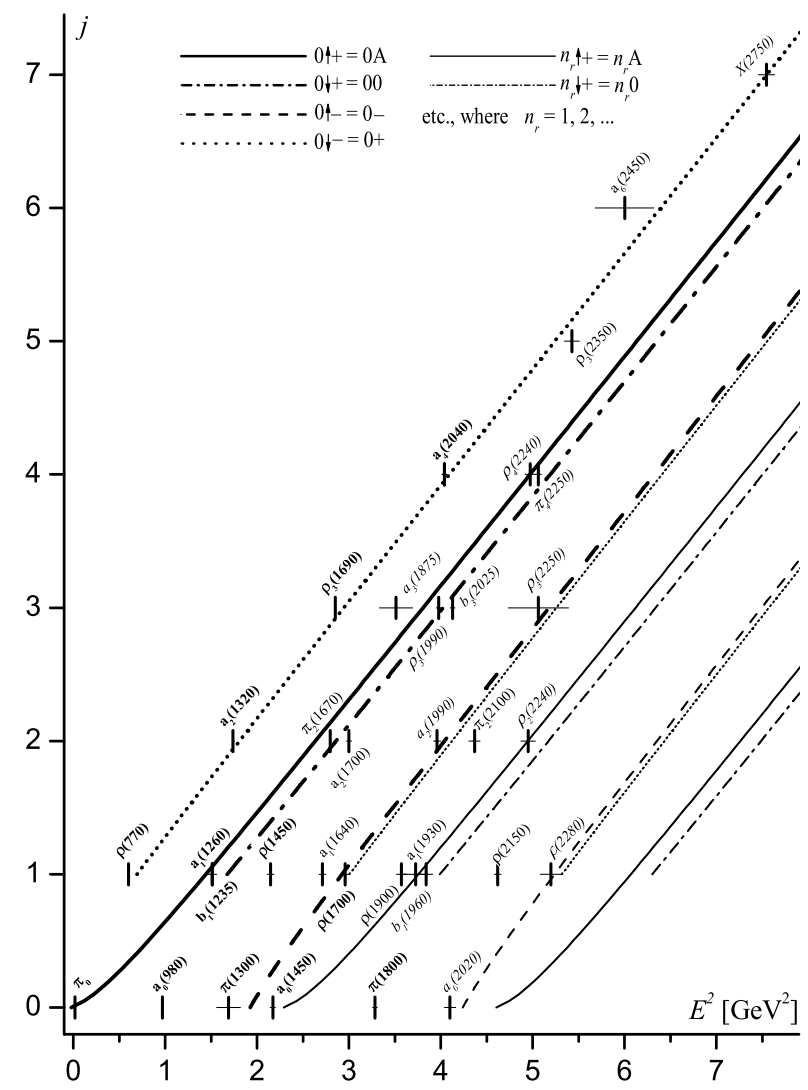

Figure 4. Spectrum of $\pi$ - and $\rho$-mesons and optimal Regge trajectories from model VI. Reliable data are inscribed in bold, doubtful - in italic. Thin horizontal lines denote measurement errors.

present case this simplicity is achieved owing to a rather nontrivial choice of the total potential

$$
\begin{aligned}
U= & \frac{\mathrm{i}}{2} \operatorname{ar} \beta_{1} \beta_{2}\left(\gamma_{1}^{5}-\gamma_{2}^{5}\right)\left(\boldsymbol{\sigma}_{1}+\boldsymbol{\sigma}_{2}\right) \cdot \boldsymbol{n}+\frac{\mathrm{i}}{4 r} \beta_{1} \beta_{2}\left(\gamma_{1}^{5}+\gamma_{2}^{5}\right)\left(\boldsymbol{\sigma}_{1}-\boldsymbol{\sigma}_{2}\right) \cdot \boldsymbol{n} \\
& +\frac{1}{2}\left(a r+\frac{1}{2 r}\right)\left(\gamma_{1}^{5}+\gamma_{2}^{5}\right)\left(\boldsymbol{\sigma}_{1} \times \boldsymbol{\sigma}_{2}\right) \cdot \boldsymbol{n} .
\end{aligned}
$$

An exact $\ell s$-degeneracy indicates the existence of additional $O(3)$-symmetry which cannot be generated by components of the total spin $\boldsymbol{s}=\frac{1}{2}\left(\boldsymbol{\sigma}_{1}+\boldsymbol{\sigma}_{2}\right)$. A search of relevant conserved quantities is beyond the scope of this work.

\section{Summary}

In this work we consider a two-body Dirac equation with general local (in the position representation) potential found by Nikitin and Fushchich in [34]. It is parameterized by 48 real functions of $r$ and is presented here in a matrix form.

Owing to $O(3)$-invariance of $2 \mathrm{BDE}$ it is reduced (via a separation of variables) to a set of eight first-order ODEs for radial components of the wave function. Then using a chain of transformations we eliminate some components of the wave function in favor of other ones and arrive at the related pair of second-order ODEs. Coefficients of these equations have, in general, poles at some energy-dependent points $r_{E}$ which are absent in an original potential. These singularities may complicate an analysis and a solution of the problem.

The structure of second-order reduction of general 2BDE suggests a wide class of potentials (parameterized by 14 arbitrary functions) for which the problem is free of non-physical singulari- 
ties. Within this class a family of exactly solvable models is found which generalize known two-particle Dirac oscillators [29, 30, 31, 32, 33]. In particular, two of these (models VI and V) are 6-parametric integrable extensions of the oscillator model [31]. Regge trajectories following from these models have parallel rectilinear asymptotes but are curved in their lover segments.

The 5-parametric model VI is used as a solvable potential model of light mesons. Special choice of parameters leads to linear Regge trajectories which possess an exact $\ell s$-degeneracy and the accidental $\left(j+2 n_{r}\right)$-degeneracy. In other words, the model restores the idealized meson spectra generating by SROM [1, 2, 3]. The corresponding two-fermion interaction potential turns out to be surprisingly intricate.

Unfortunately, the model fails to describe properly the spectrum of lightest mesons as it overestimates meson masses squared $M^{2}$ by certain (common for all states) constant $c^{2}$. Up to this discrepancy the description of $(\pi-\rho)$-family is adequate and has been realized. The choice of parameters in this case differs slightly from that of the completely degenerated model so that the Regge trajectories are somewhat curved in the bottom. The fit is good for most of orbital meson excitations and worse - for radial ones due to shortage of daughters following from the model. It follows from this fact that the accidental $\left(j+2 n_{r}\right)$-degeneracy inherent approximately or exactly in this and many other models $[1,2,3,40]$ is less adequate to actual meson data than the degeneracy of $\left(j+n_{r}\right)$-type.

Despite the 2BDE used in this work being $O(3)$-invariant and not truly relativistic equation, it can be considered as some covariant equation reduced in the center-of-mass frame of reference. In Appendix we constructed explicitly the Poincaré-invariant equation for 2BDE with potential of general form and proved its unambiguity.

\section{Appendix. Covariant form of 2BDE}

The two body Dirac equation considered in this work is $O(3)$-invariant but not Poincaréinvariant. Below we construct the manifestly covariant equations reduction of which in the center-of-mass reference frame restores the 2BDE (1) with arbitrary potential of the form (3).

We start with a free-particle system. Following by the constraint formalism $[10,11,12,13$, $14,15]$ it is described by the pair of covariant Dirac equations

$$
\left(\gamma_{a} \cdot p_{a}-m_{a}\right) \Phi=0, \quad a=1,2
$$

where $\gamma_{a} \cdot p_{a} \equiv \gamma_{a}^{\mu} p_{a \mu}$, the particle 4-momenta $p_{a \mu}(a=1,2 ; \mu=0, \ldots, 3)$ are conjugated to particle positions $x_{a}^{\mu}$, and the timelike Lorentz metrics $\left\|\eta_{\mu \nu}\right\|=\operatorname{diag}(+,-,-,-)$ is used.

Before introducing an interaction it is convenient to represent the equations (A.1) in a collective form. For this purpose we perform the canonical transformation $\left(x_{a}^{\mu}, p_{a \mu}\right) \rightarrow\left(X^{\mu}, P_{\mu}, x^{\mu}, p_{\mu}\right)$

$$
\begin{aligned}
& x=x_{1}-x_{2}, \quad P=p_{1}+p_{2}, \quad M=\sqrt{P^{2}}, \\
& p=p_{1}-\xi(M) P, \quad X=\xi x_{1}+(1-\xi) x_{2}+\frac{d \xi}{d M}(P \cdot x) \hat{P}, \quad \hat{P}=P / M,
\end{aligned}
$$

where $\xi(M)$ is an arbitrary function, and introduce the operators

$$
\begin{aligned}
\mathcal{H}^{\text {free }}=\gamma_{2}^{\prime \prime}\left(\gamma_{1} \cdot p_{1}-m_{1}\right)+\gamma_{1}^{\prime \prime}\left(\gamma_{2} \cdot p_{2}-m_{2}\right) \\
\quad=\gamma_{1}^{\prime \prime} \gamma_{2}^{\prime \prime} M+\gamma_{2}^{\prime \prime}\left(\gamma_{1} \cdot p-m_{1}\right)+\gamma_{1}^{\prime \prime}\left(-\gamma_{2} \cdot p-m_{2}\right), \\
\mathcal{K}=\frac{1}{2}\left(\gamma_{1} \cdot p_{1}+m_{1}\right)\left(\gamma_{1} \cdot p_{1}-m_{1}\right)-\frac{1}{2}\left(\gamma_{2} \cdot p_{2}+m_{2}\right)\left(\gamma_{2} \cdot p_{2}-m_{2}\right) \\
=\frac{1}{2}\left(p_{1}^{2}-p_{2}^{2}-m_{1}^{2}+m_{2}^{2}\right)=P \cdot p-\nu(M),
\end{aligned}
$$

such that $\left[\mathcal{H}^{\text {free }}, \mathcal{K}\right]=0$; here $\gamma_{a}^{\prime \prime} \equiv \gamma_{a} \cdot \hat{P}$ and $\nu(M)=\frac{1}{2}\left(m_{1}^{2}-m_{2}^{2}+(1-\xi(M)) M^{2}\right)$. In these terms the equations (A.1) take the equivalent form $\mathcal{H}^{\text {free }} \Phi=0$ and $\mathcal{K} \Phi=0$. 
At this point we choose the mixed representation for the wave function: $\Phi=\Phi(P, x)$. Then it follows from the constraint $\mathcal{K} \Phi=0$

$$
\Phi(P, x)=\exp \left(\mathrm{i} \frac{\nu(M)}{M} x^{\prime \prime}\right) \tilde{\Phi}\left(P, x_{\perp}\right),
$$

where $x^{\prime \prime}=\hat{P} \cdot x \stackrel{\boldsymbol{P}=0}{\longrightarrow} x^{0}$ and $x_{\perp}^{\mu}=\left(\delta_{\nu}^{\mu}-\hat{P}^{\mu} \hat{P}_{\nu}\right) x^{\nu} \stackrel{\boldsymbol{P}=0}{\longrightarrow}(0, \boldsymbol{r})$ thus $\tilde{\Phi}\left(P, x_{\perp}\right) \stackrel{\boldsymbol{P}=0}{\longrightarrow} \tilde{\Phi}(E, \boldsymbol{r})$ where $E=\left.P_{0}\right|_{\boldsymbol{P}=0}=\left.M\right|_{\boldsymbol{P}=0}$. In other words, this constraint suppresses the $x^{\prime \prime}$-dependence of $\tilde{\Phi}$ and eliminates the relative time $x^{0}$ from the center-of-mass description. Equivalently, one can choose $\nu(M)=0$, then $\Phi$ is free of $x^{\prime \prime}$. We note that the choice of $\nu(M)$ or $\xi(M)$ does not affect the form of the operator $\mathcal{H}^{\text {free }}$ and so it is gauge-fixing. Thus we do not distinguish the functions $\Phi$ and $\tilde{\Phi}$ any more.

In the center-of-mass (c.m.) frame of reference (where $\boldsymbol{P}=0$ ) we have

$$
\mathcal{H}^{\text {free }} \Phi\left(P, x_{\perp}\right) \stackrel{\boldsymbol{P}=0}{\longrightarrow} \beta_{1} \beta_{2}\left\{E-h_{1}(\boldsymbol{p})-h_{2}(-\boldsymbol{p})\right\} \Phi(\boldsymbol{r})=0,
$$

where $\boldsymbol{p}=\boldsymbol{p}_{1}=-\boldsymbol{p}_{2}, \beta_{a} \equiv \gamma_{a}^{0}=\left.\gamma^{\prime \prime}\right|_{\boldsymbol{P}=0}$, and operators $h_{a}(\boldsymbol{p})$ are defined in (2). Up to the factor $-\beta_{1} \beta_{2}$ this equation coincides with $2 \mathrm{BDE}$ (1) in the case of free particles.

In the general case the interaction term $\mathcal{V} \Phi$ appears in the r.h.s. of the equation $\mathcal{H}^{\text {free }} \Phi=0$ (instead of zero), and we have the following set of equations

$$
P \cdot p \Psi=0 \quad \text { and } \quad \mathcal{H}^{\text {free }} \Psi=\mathcal{V} \Psi .
$$

Since a free-particle operator $\mathcal{H}^{\text {free }}$ is Poincaré-invariant the potential $\mathcal{V}$ must be so. Besides, it must obey the equalities

$$
\left.\mathcal{V}_{\text {c.m. }} \equiv \mathcal{V}\right|_{\boldsymbol{P}=0}=\beta_{1} \beta_{2} U(\boldsymbol{r})
$$

and $[\mathcal{V}, P \cdot p]=0$. These requirements allow us to construct unambiguously the covariant operator $\mathcal{V}$ for arbitrary potential of the form (3).

It follows from (A.2), (3) that the operator $\mathcal{V}_{\text {c.m. }}$ consists of the sum of partial potentials $U_{A}(r)$ multiplied by matrix coefficients $\beta_{1} \beta_{2} \Gamma_{A}$. Both factors in every term of the sum are $O(3)$ invariant. We construct Lorentz-scalar counterparts for them separately.

Let $\rho=\sqrt{-x_{\perp}^{2}}$ and thus $\rho_{\text {c.m. }}=|\boldsymbol{r}|=r$. Then functions $U_{A}(\rho)$ are Lorentz scalars by construction and $\vec{U}_{A}(\rho)_{\text {c.m. }}=U_{A}(r)$.

(5)-(6) is a basis for matrices $\beta_{1} \beta_{2} \Gamma_{A}: \beta_{1} \beta_{2} \Gamma_{\text {ee }}=\Gamma_{\text {ee }}, \beta_{1} \beta_{2} \Gamma_{\text {oo }}=\Gamma_{\text {oo }}, \beta_{1} \beta_{2} \Gamma_{\text {eo }}=\mathrm{i} \Gamma_{\text {eo }}$, $\beta_{1} \beta_{2} \Gamma_{\mathrm{oe}}=\mathrm{i} \Gamma_{\mathrm{oe}}$ (the factor "i" is not important in this regard). First of all it is convenient to express $\boldsymbol{\sigma}$-matrices in the spin factors $(7),(8)$ of the basis (5)-(6) via $\boldsymbol{\gamma}$-matrices as follows: $\boldsymbol{\sigma}_{a}=\gamma_{a}^{5} \beta_{a} \gamma_{a}$. Then every basis element is a product of factors

$$
I, \quad \gamma_{a}^{5}, \quad \beta_{a}, \quad \gamma_{1} \cdot \gamma_{2}, \quad \gamma_{a} \cdot \boldsymbol{n}, \quad\left(\boldsymbol{n}, \gamma_{1}, \gamma_{2}\right)
$$

which are scalar or pseudo-scalar with respect to the group $O(3)$. We construct the Lorentz scalar or pseudo-scalar counterparts of these matrices as follows

$$
\begin{aligned}
& I \rightarrow I, \quad \gamma_{a}^{5} \rightarrow \gamma_{a}^{5}, \quad \beta_{a} \rightarrow \gamma_{a}^{\prime \prime}, \quad \gamma_{1} \cdot \gamma_{2} \rightarrow-\gamma_{1}^{\perp} \cdot \gamma_{2}, \\
& \gamma_{a} \cdot \boldsymbol{n} \rightarrow-\gamma_{a} \cdot n_{\perp}, \quad\left(\boldsymbol{n}, \gamma_{1}, \gamma_{2}\right) \rightarrow-\left(\hat{P}, n, \gamma_{1}, \gamma_{2}\right) \equiv-\varepsilon_{\mu \nu \lambda \varkappa} \hat{P}^{\mu} n^{\nu} \gamma_{1}^{\lambda} \gamma_{2}^{\varkappa},
\end{aligned}
$$

where $n=x / \rho$ and $\varepsilon_{\mu \nu \lambda \varkappa}$ is absolutely antisymmetric pseudo-tensor $\left(\varepsilon_{0123}=-1\right)$.

The resulting covariant potential $\mathcal{V}$ has the form

$$
\mathcal{V}(x, \hat{P})=\sum_{A=1}^{48} U_{A}(\rho) \Xi_{A},
$$

where matrices $\Xi_{A}$ are built with $\beta_{1} \beta_{2} \Gamma_{A}$ by the replacement (A.3) (with an unchanged order of co-factors).

The unambiguity of this construction is obvious: if there exist two potentials which coincide in the c.m. reference frame then their difference is zero in any reference frame, due to Poincaréinvariance of these potentials. 


\section{References}

[1] Kim Y.S., Noz M.E., Covariant harmonic oscillator and the quark model, Phys. Rev. D 8 (1973), 3521-3527.

[2] Takabayasi T., Relativistic mechanics of confined particles as extended model of hadrons, Progr. Theoret. Phys. Suppl. 67 (1979), 1-68.

[3] Ishida S., Oda M., A universal spring and meson orbital Regge trajectories, Nuovo Cimento A 107 (1994), 2519-2525.

[4] Barut A.O., Komy S., Derivation of nonperturbative relativistic two-body equation from the action principle in quantum electrodynamics, Fortschr. Phys. 33 (1985), 309-318.

[5] Barut A.O., Ünal N., A new approach to bound-state quantum electrodynamics, Phys. A 142 (1987), 467-487.

[6] Grandy W.T. Jr., Relativistic quantum mechanics of leptons and fields, Kluwer Academic Publishers, Dordrecht - Boston - London, 1991.

[7] Duviryak A., Large- $j$ expansion method for two-body Dirac equation, SIGMA 2 (2006), 029, 12 pages, math-ph/0602066.

[8] Breit G., The effect of retardation on the interaction of two electrons, Phys. Rev. 34 (1929), 553-573.

[9] Bethe H.A., Salpeter E.E., Quantum mechanics of one- and two-electron atoms, Springer-Verlag, Berlin Göttingen - Heidelberg, 1957.

[10] Sazdjian H., Relativistic wave equations for the dynamics of two interacting particles, Phys. Rev. D 33 (1986), 3401-3424.

[11] Mourad J., Sazdjian H., The two-fermion relativistic wave equations of constraint theory in the PauliSchrödinger form, J. Math. Phys. 35 (1994), 6379-6406, hep-ph/9403232.

[12] Mourad J., Sazdjian H., How to obtain a covariant Breit type equation from relativistic constraint theory, J. Phys. G 21 (1995), 267-279, hep-ph/9412261.

[13] Crater H.W., Van Alstine P., Two-body Dirac equations for particles interacting through world scalar and vector potentials, Phys. Rev. D 36 (1987), 3007-3035.

[14] Crater H.W., Van Alstine P., Extension of two-body Dirac equations to general covariant interactions through a hyperbolic transformation, J. Math. Phys. 31 (1990), 1998-2014.

[15] Crater H.W., Van Alstine P., Two-body Dirac equations for relativistic bound states of quantum field theory, hep-ph/9912386.

[16] Krolikowski W., Relativistic radial equations for 2 spin-1/2 particles with a static interaction, Acta Phys. Polon. B 7 (1976), 487-496.

[17] McClary R., Byers N., Relativistic effects in heavy-quarkonium spectroscopy, Phys. Rev. D 28 (1983), 1692-1705.

[18] Crater H.W., Van Alstine P., Relativistic naive quark model for spining quarks in mesons, Phys. Rev. Lett. 53 (1984), 1527-1530.

[19] Crater H.W., Van Alstine P., Relativistic constraint dynamics for spining quarks in mesons, in Constraint's Theory and Relativistic Dynamics, Editors G. Longhi and L. Lusanna, World Scientific Publ., Singapore, 1987, 210-241.

[20] Childers R.W., Effective Hamiltonians for generalized Breit interactions in QCD, Phys. Rev. D 36 (1987), 606-614.

[21] Brayshaw D.D., Relativistic description of quarkonium, Phys. Rev. D 36 (1987), 1465-1478.

[22] Ceuleneer R., Legros P., Semay C., On the connection between relativistic and nonrelativistic description of quarkonium, Nuclear Phys. A 532 (1991), 395-400.

[23] Semay C., Ceuleneer R., Silvestre-Brac B., Two-body Dirac equation with diagonal central potentials, J. Math. Phys. 34 (1993), 2215-2225.

[24] Semay C., Ceuleneer R., Two-body Dirac equation and Regge trajectories, Phys. Rev. D 48 (1993), 43614369.

[25] Tsibidis G.D., Quark-antiquark bound states and the Breit equation, Acta Phys. Polon. B 35 (2004), $2329-$ 2366, hep-ph/0007143.

[26] Moshinsky M., Nikitin A.G., The many body problem in relativistic quantum mechanics, Rev. Mexicana de Fís. 50 (2005), 66-73, hep-ph/0502028. 
[27] Crater H.W., Van Alstine P., Relativistic calculation of the meson spectrum: a fully covariant treatment versus standard treatments, Phys. Rev. D 70 (2004), 034026, 31 pages, hep-ph/0208186.

[28] Duviryak A., Application of two-body Dirac equation in meson spectroscopy, J. Phys. Stud. 10 (2006), 290-314 (in Ukrainian).

[29] Sazdjian H., Relativistic quarkonium dynamics, Phys. Rev. D 33 (1986), 3425-3434.

[30] Sazdjian H., Supersymmetric models in two-particle relativistic quantum mechanics, Eur. Lett. 6 (1988), $13-18$.

[31] Moshinsky M., Loyola G., Villegas C., Anomalous basis for representations of the Poincaré group, J. Math. Phys. 32 (1991) 32, 373-381.

[32] Moshinsky M., Del Sol Mesa A., Relations between different approaches to the relativistic two-body problem, J. Phys. A: Math. Gen. 27 (1994), 4684-4693.

[33] Moshinsky M., Quesne C., Smirnov Yu.F., Supersymmetry and superalgebra for the two-body system with a Dirac oscillator interaction, J. Phys. A: Math. Gen. 28 (1995), 6447-6457, hep-th/9510006.

[34] Nikitin A.G., Fushchich W. I., Non-Lie integrals of the motion for particles of arbitrary spin and for systems of interacting particles, Teoret. Mat. Fiz. 88 (1991), 406-515 (English transl.: Theoret. and Math. Phys. 88 (1991), 960-967).

[35] Darewych J.W., Duviryak A., Exact few-particle eigenstates in partially reduced QED, Phys. Rev. A 66 (2002), 032102, 20 pages, nucl-th/0204006.

[36] Chraplyvy Z.V., Reduction of relativistic two-particle wave equations to approximate forms. I, Phys. Rev. 91 (1953), 388-391.

[37] Messiah A., Quantum mechanics, Vol. 2, Willey, New York, 1961.

[38] Berdnikov E.B., Pronko G.P., Relativistic model of orbital excitations of mesons, J. Nuclear Phys. 54 (1991), 763-776.

[39] Goebel C., LaCourse D., Olsson M.G., Systematics of some ultrarelativistic potential models, Phys. Rev. D 41 (1990), 2917-2923.

[40] Simonov Yu.A., Ideas in nonperturbative QCD, Nuovo Cimento A 107 (1994), 2629-2644, hep-ph/9311217.

[41] Khruschev V.V., Mass formula for mesons containing light quarks, Preprint IHEP 87-9, Serpukhov, 1987.

[42] Duviryak A., The two-particle time-asymmetric relativistic model with confinement interaction and quantization, Internat. J. Modern Phys. A 16 (2001), 2771-2788.

[43] Inopin A., Sharov G.S., Hadronic Regge trajectories: problems and approaches, Phys. Rev. D 63 (2001), 054023, 10 pages, hep-ph/9905499.

[44] Borodulin V.I., Plyushchay M.S., Pron'ko G.P., Relativistic string model of light mesons with massless quarks, Z. Phys. C 41 (1988), 293-302.

[45] Ishida S., Yamada K., Light-quark meson spectrum in the covariant oscillator quark model with one-gluonexchange effects, Phys. Rev. D 35 (1987), 265-281.

[46] Yao W.-M. et al., The review of particle physics, J. Phys. G 33 (2006), 1-1232. 